\title{
THE BUCHBERGER RESOLUTION
}

\author{
ANDA OLTEANU AND VOLKMAR WELKER
}

\begin{abstract}
We define the Buchberger resolution, which is a graded free resolution of a monomial ideal in a polynomial ring. Its construction uses a generalization of the Buchberger graph and encodes much of the combinatorics of the Buchberger algorithm. The Buchberger resolution is a cellular resolution that, when it is minimal, coincides with the Scarf resolution. The simplicial complex underlying the Buchberger resolution is of interest for its own sake, and its combinatorics is not fully understood. We close with a conjecture on the clique complex of the Buchberger graph.
\end{abstract}

Introduction. Let $S=\mathbb{k}\left[x_{1}, \ldots, x_{n}\right]$ be the polynomial ring in $n$ variables over a field $\mathbb{k}$ and $I$ a monomial ideal of $S$. The construction of the minimal graded free resolution of $I$ over $S$ is an important problem in commutative algebra. Even though there are good algorithms that construct the minimal graded free resolution for general monomial ideals, there is no known combinatorial construction of the minimal free graded resolution. In this paper, we provide a construction of a new free resolution for all monomial ideals and then identify the ideals for which it is minimal. We call this resolution the Buchberger resolution since its combinatorics is derived from the Buchberger algorithm, an idea first employed in the three variable case [5]. The Buchberger resolution is a cellular resolution. Roughly speaking, a cellular resolution is given by simplicial complex (or, more generally, CW-complex) with a labelling of its vertices by generators of a monomial ideal and an induced labelling of its simplices by the lcms of their vertices. If the conditions from [1, Lemma 2.2] are fulfilled, then the homogenized simplicial chain

2010 AMS Mathematics subject classification. Primary 05C10, 13C14, 13D02.

Keywords and phrases. Monomial resolution, cellular resolution, Scarf complex, minimal free resolution.

The first author was supported by the Romanian Ministry of Education, CNCSUEFISCDI, project No. PN-II-RU-PD-2012-3-0235. The second author was partially supported by MSRI.

Received by the editors on October 12, 2014, and in revised form on May 7, 2015. 
complex of the simplicial complex with coefficients in the polynomial ring supports a free resolution of the ideal.

One prominent example of a cellular resolution is the Taylor resolution [10] which is defined for arbitrary monomial ideals and supported by the full simplex on the set $\mathcal{G}(I)$ of minimal monomial generators of $I$; rarely, the Taylor resolution is minimal. In [1, Theorem 3.2], it is shown that there is a subcomplex of the simplex on $\mathcal{G}(I)$, the Scarf complex, which, for example, supports a minimal free resolution for generic monomial ideals, but that it does not even support a free resolution in general. In [5], the Buchberger graph of a monomial ideal is studied and, in the case of three variables, its planar embeddings are used to define a simplicial complex supporting a minimal free resolution for strongly generic ideals. Our Buchberger resolution will be supported on a simplicial complex that generalizes the Scarf complex and the planar map of a Buchberger graph. It is shown to coincide with the Scarf complex and the planar map of the Buchberger graph in cases where the Buchberger resolution is minimal. For references to other constructions of cellular resolutions, we refer the reader to [7].

The paper is organized as follows. In Section 1, we fix the notation and recall the concept of the Buchberger graph associated to a monomial ideal from [5]. Then, we define the Buchberger complex and show that it is a contractible simplicial complex. Therefore, by inductive reasoning and [1, Lemma 2.2], it supports a graded free resolution, which we call the Buchberger resolution. Section 2 is devoted to the relation between the Buchberger resolution and the Scarf complex. The Buchberger resolution turns out to be minimal precisely when the Scarf complex is a resolution.

\section{Cellular resolutions, the Buchberger graph and the Buch-} berger complex. In this section, we associate the Buchberger complex to any monomial ideal, the vertices of which are labeled by the minimal monomial generators (1.7), and we show that it supports a resolution. For the case of monomial ideals which are not squarefree, this simplicial complex is "smaller" than the Taylor complex, see Example 1.9. Therefore, the resolution obtained will be smaller than the Taylor resolution but still in general not minimal. 
We begin by recalling the notion of a cellular resolution of a monomial ideal $I$ in the polynomial ring

$$
S=\mathbb{k}\left[x_{1}, \ldots, x_{n}\right]
$$

over a field $\mathbb{k}$ in its original definition [1].

Let $\Delta$ be a simplicial complex whose vertices are bijectively labeled by the monomials from the set of minimal monomial generators $\mathcal{G}(I)$ of $I$. Each face $F \in \Delta$ is labeled by the least common multiple of its vertices, which we denote by $\mathbf{m}_{F}$. The multidegrees of these monomials define an $\mathbb{N}^{n}$-grading of $\Delta$. Let $\mathcal{F}_{\Delta}$ be the $\mathbb{N}^{n}$-graded chain complex of $\Delta$ over $S$ with differentials homogenized with respect to the grading (see [1] for details).

Proposition 1.1 ([1, Lemma 2.2]). The complex $\mathcal{F}_{\Delta}$ is exact and defines a free resolution of $I$ if and only if, for every monomial $m$, the simplicial complex

$$
\Delta[m]=\left\{F \in \Delta \mid \mathbf{m}_{F} \text { divides } m\right\}
$$

is empty or acyclic over $\mathbb{k}$.

If the complex $\mathcal{F}_{\Delta}$ is exact, then it is called the resolution supported by the (labeled) simplicial complex $\Delta$. Moreover, if $\mathcal{F}_{\Delta}$ is exact, one can determine whether or not it is a minimal free resolution of $I$.

Proposition $1.2\left(\left[2\right.\right.$, Remark 1.4]). Let $\mathcal{F}_{\Delta}$ be a free resolution of the monomial ideal I supported by the labeled simplicial complex $\Delta$. Then $\mathcal{F}_{\Delta}$ is a minimal free resolution if and only if any two comparable faces $F \subset G$ of the complex $\Delta$ have distinct degrees, that is $\mathbf{m}_{F} \neq \mathbf{m}_{G}$.

Next, we describe the construction of the Buchberger graph [6] and its connection to minimal free resolutions.

We define a partial order $<$ on $\mathbb{N}^{n}$ as follows. For $\mathbf{a}, \mathbf{b} \in \mathbb{N}^{n}$, where

$$
\begin{aligned}
& \mathbf{a}=\left(a_{1}, \ldots, a_{n}\right), \\
& \mathbf{b}=\left(b_{1}, \ldots, b_{n}\right) .
\end{aligned}
$$


One says that $\mathbf{a}<\mathbf{b}$ if $a_{i}<b_{i}$ for all $1 \leq i \leq n$ such that $b_{i} \neq 0$ and $a_{i}=b_{i}$ if $b_{i}=0$. Given a monomial,

$$
u=x_{1}^{u_{1}} \cdots x_{n}^{u_{n}} \quad \text { in } S,
$$

we denote its multidegree by $\operatorname{mdeg}(u)$, that is, $\operatorname{mdeg}(u)=\left(u_{1}, \ldots, u_{n}\right)$. For two monomials $u$ and $v \in S$, one says that $u$ properly divides $v$ if $u$ divides $v$ and $\operatorname{mdeg}(u)<\operatorname{mdeg}(v)$. We will denote this by $\left.u\right|_{p} v$.

In [5], Miller and Sturmfels associate to any monomial ideal its Buchberger graph which first appeared under this name in [6].

Definition 1.3. The Buchberger graph of the monomial ideal $I$, denoted $\mathfrak{B u G}(I)$, is the graph on vertex set $\mathcal{G}(I)$ with edges $F=\left\{m, m^{\prime}\right\}$ for distinct monomials $m, m^{\prime} \in \mathcal{G}(I)$ such that there is no monomial $m^{\prime \prime} \in \mathcal{G}(I)$ which properly divides the least common multiple $\mathbf{m}_{F}$.

The Buchberger graph plays an important role in Gröbner basis theory [6] and also appears in the study of special classes of monomial ideals such as strongly generic ideals.

Definition 1.4. A monomial ideal $I \subseteq S$ is called strongly generic if, for any two monomials $u, v \in \mathcal{G}(I)$,

$$
u=x_{1}^{u_{1}} \cdots x_{n}^{u_{n}}
$$

and

$$
v=x_{1}^{v_{1}} \cdots x_{n}^{v_{n}},
$$

the condition $\left(u_{i} \neq v_{i}\right.$ or $u_{i}=v_{i}=0$ for all $\left.1 \leq i \leq n\right)$ is fulfilled.

In the case of polynomial rings in three variables, there is a connection between the minimal graded free resolution of strongly generic ideals and their corresponding Buchberger graphs.

Proposition 1.5 ([6, Proposition 3.9]). Let $I \subseteq \mathbb{k}[x, y, z]$ be a strongly generic ideal. Then $\mathfrak{B u G}(I)$ is planar and connected.

A planar graph $G$, together with an (sufficiently nice) embedding of $G$ into the plane $\mathbb{R}^{2}$ is called a planar map. The vertices of the 
embedded graph can be considered as 0-cells, the edges as 1-cells and the regions bounded by $G$ as 2-cells. Viewed from this perspective, the embedding defines a CW-complex. In [6, page 51], it is shown that this complex supports a free resolution of some monomial ideal. Moreover:

Theorem 1.6 ([6, Theorem 3.11]). Let $I \subseteq \mathbb{k}[x, y, z]$ be a strongly generic ideal. Then any planar map of $\mathfrak{B u G}(I)$ with vertices labeled by the generators and edges and faces labeled by the lcms of its vertices supports a minimal free resolution of $I$.

It is natural to ask: Can this result be extended to strongly generic ideals in a polynomial ring in $n$ variables, $S=\mathbb{k}\left[x_{1}, \ldots, x_{n}\right]$ ?

In order to answer this question, we generalize the Buchberger graph to a suitable simplicial complex and then prove that this simplicial complex supports a free resolution.

Definition 1.7. Let $I \subseteq \mathbb{k}\left[x_{1}, \ldots, x_{n}\right]$ be a monomial ideal. The Buchberger complex $\mathfrak{B u}(I)$ of $I$, is the collection of all subsets $F$ of $\mathcal{G}(I)$ such that no $u \in \mathcal{G}(I)$ properly divides $\mathbf{m}_{F}$.

The set system $\mathfrak{B} \mathfrak{u}(I)$ is indeed a simplicial complex. To see this, assume that $F \in \mathfrak{B u}(I)$ and $G \subsetneq F, G \notin \mathfrak{B u}(I)$. Then $\mathbf{m}_{G} \mid \mathbf{m}_{F}$. But $G \notin \mathfrak{B u}(I)$, and hence, there is a minimal monomial generator $u \in I$ such that $\left.u\right|_{p} \mathbf{m}_{G}$, which implies $\left.u\right|_{p} \mathbf{m}_{F}$, a contradiction.

\section{Remark 1.8.}

- The 1-skeleton $\mathfrak{B u}(I)^{\langle 1\rangle}$ of the Buchberger complex $\mathfrak{B u}(I)$ of the monomial ideal $I$ is the Buchberger graph $\mathfrak{B} \mathfrak{u} \mathfrak{G}(I)$ of $I$.

- If $I$ is a squarefree monomial ideal, then its Buchberger complex is the full simplex $2^{\mathcal{G}(I)}$.

Example 1.9. Let

$$
I=\left(x_{1}^{2}, x_{2}^{2}, x_{3}^{2}, x_{1} x_{3}, x_{2} x_{4}\right) \subseteq \mathbb{k}\left[x_{1}, x_{2}, x_{3}, x_{4}\right] .
$$

It is easy to see that $\left.x_{1} x_{3}\right|_{p} x_{1}^{2} x_{3}^{2}=\operatorname{lcm}\left(x_{1}^{2}, x_{3}^{2}\right)$; therefore, $\left\{x_{1}^{2}, x_{3}^{2}\right\} \notin$ $\mathfrak{B u}(I)$. In this case, the Buchberger complex has two facets (see 
Figure 1), namely,

$$
\mathfrak{B u}(I)=\left\langle\left\{x_{1}^{2}, x_{2}^{2}, x_{1} x_{3}, x_{2} x_{4}\right\},\left\{x_{3}^{2}, x_{2}^{2}, x_{1} x_{3}, x_{2} x_{4}\right\}\right\rangle .
$$

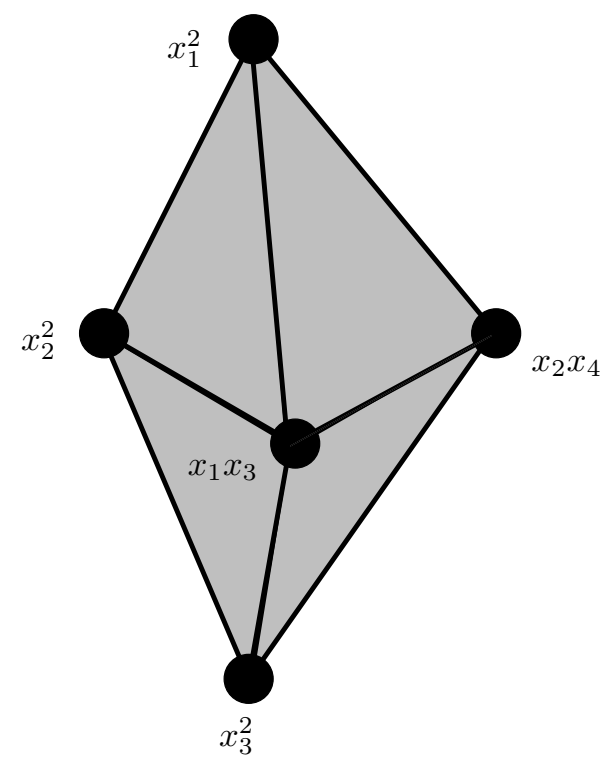

FiguRE 1. Buchberger complex of $I=\left(x_{1}^{2}, x_{2}^{2}, x_{3}^{2}, x_{1} x_{3}, x_{2} x_{4}\right)$.

In order to verify the conditions from Proposition 1.1 for the Buchberger complex, we must introduce some combinatorial constructions and exhibit some of their properties.

Let $P$ be a finite poset and $\Delta(P)$ its order complex, that is, the simplicial complex whose simplices are the chains in the poset $P$. Through the geometric realization of $\Delta(P)$, we then can speak of homotopy type, homotopy equivalence and contractability of posets (see [12]). We associate to a monomial ideal $I$ its lcm-lattice $L_{I}$, which is the poset on the least common multiples $\mathbf{m}_{F}$ of subsets $F \subseteq \mathcal{G}(I)$ ordered by divisibility [4]. It is easily checked that $L_{I}$ indeed is a lattice with least element $1=\operatorname{lcm}(\emptyset)$. For a monomial $m \in L_{I}$, we write $(1, m)$ for the open interval of all $m^{\prime} \in L_{I}$ such that $1<m^{\prime}<m$. 
In the next two lemmas we collect basic topological properties of the lcm lattice that will prove crucial for our first main result.

Lemma 1.10. Let $m \in L_{I}$. If there is a monomial $w \in \mathcal{G}(I)$ such that $\left.w\right|_{p} m$, then the interval $(1, m)$ is contractible.

Proof. Let $w \in \mathcal{G}(I)$ be a monomial which properly divides $m$. We consider the map $f:(1, m) \rightarrow(1, m)$, defined by $f(u)=\operatorname{lcm}(u, w)$. Since $w$ properly divides $m$, it follows that $f(u) \in(1, m)$ for all $u \in(1, m)$. By construction for $u, u^{\prime} \in(1, m)$ and $u \leq u^{\prime}$ it follows that $f(u) \leq f\left(u^{\prime}\right)$. Again, by definition, $f(f(u))=f(u)$, and hence, $f$ is a closure operator on the poset $(1, m)$. By [3, Corollary 10.11] this shows that the interval $(1, m)$ and its image under $f$ are homotopy equivalent. Moreover, the image of $(1, m)$ under $f$ has $w$ as its unique smallest element. Therefore, the order complex of the image of $f$ is a cone over $w$, and hence, contractible. Thus, $(1, m)$ is contractible itself.

Lemma 1.11. Let $P_{I}$ be the poset of all monomials $m \in L_{I} \backslash\{1\}$ such that there is no monomial $w \in \mathcal{G}(I)$ which properly divides $m$. Then $P_{I}$ is contractible.

Proof. By Lemma 1.10, we know that, for each $m \in L_{I}$ for which there is a $w \in \mathcal{G}(I)$ that properly divides $m$, the interval $(1, m)$ is contractible. Let $M$ be the set of all $m \in L_{I}$ with this property. Then a simple application of the Quillen fiber lemma (see [3, Theorem 10.11]) shows that

$$
L_{I} \backslash(M \cup\{1\})
$$

is homotopy equivalent to $L_{I} \backslash\{1\}$. But $L_{I} \backslash\{1\}$ has $\operatorname{lcm} \mathcal{G}(I)$ as its unique maximal element, and hence, its geometric realization is a cone. Therefore, it is contractible. But this shows that $P_{I}$ must be contractible.

For the proof of our first main theorem we need yet another concept from topological combinatorics. We call a subset $B$ of a poset $P$ bounded if there are elements $m$ and $m^{\prime}$ in $P$ such that $m \leq n \leq m^{\prime}$ for all $n \in B$. A poset $P$ is called an antichain if any two elements of $P$ are incomparable. A crosscut $A$ in $P$ is an antichain such that, 
for every chain $C \subseteq P$, there exists an element $a \in A$ such that $a$ is comparable with all elements in $C$, and every bounded subset $B$ of $A$ has an infimum and a supremum. Let $\Gamma(P, A)$ be the collection of subsets of $A$ that are bounded. It is easily seen that $\Gamma(P, A)$ is a simplicial complex. It is called the crosscut complex of $P$ and $A$.

Example 1.12. We consider the poset $P$ on the ground set $\{1, \ldots, 7\}$, whose Hasse diagram is represented in Figure 2. The set $A=\{3,4,5\}$ is a crosscut in $P$. Then the crosscut complex of $P$ and $A$ is

$$
\Gamma(P, A)=\langle\{3,4\},\{4,5\}\rangle .
$$

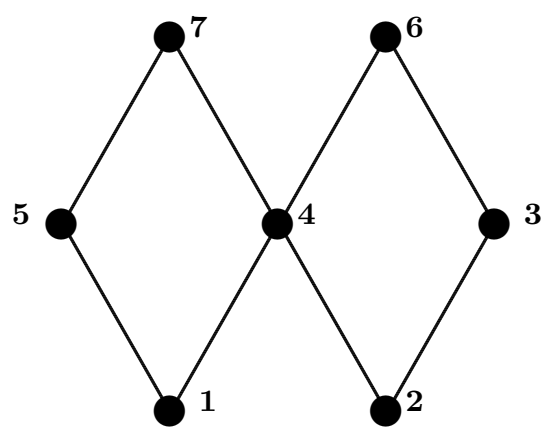

FIGURE 2.

The relation between the crosscut complex $\Gamma(P, A)$ and the topology of $P$ is given by the homotopy version of Rota's crosscut theorem, which says that (see [3, Theorem 10.8]) for a finite poset $P$ and a crosscut $A$ in $P$, the complexes $\Gamma(P, A)$ and $\Delta(P)$ are homotopy equivalent.

Theorem 1.13. Let $I$ be a monomial ideal. Then the Buchberger complex $\mathfrak{B u}(I)$ supports a cellular resolution of $I$.

Proof. We will first verify that $\mathfrak{B u}(I)$ is contractible and then show that this implies the criterion from Proposition 1.1 for a simplicial complex to support a free resolution.

Let $P_{I}$ be the poset of all monomials $m \in L_{I} \backslash\{1\}$ such that there is no monomial $w \in \mathcal{G}(I)$ which properly divides $m$. If $m \in P_{I}$ and 
$m^{\prime} \in L_{I}$ divides $m$, then $m^{\prime} \in P_{I}$. Otherwise, there is $m^{\prime \prime} \in L_{I}$ which properly divides $m^{\prime}$, and hence, properly divides $m$, contradicting $m \in P_{I}$. Thus, $P_{I}$ is a lower order ideal in $L_{I} \backslash\{1\}$. As $L_{I}$ is a lattice this implies that any subset of $P_{I}$ which is bounded from below (respectively, above) has an infimum (respectively, supremum) in $P_{I}$.

Let $A=\mathcal{G}(I)$, so $A \subseteq P_{I}$. Since $A$ is a generating set of the monomial ideal $I$, for every chain in $P_{I}$, there is an element from $A$ comparable with all elements from the chain. Moreover, $A$ is an antichain due to the fact that any two monomials from the minimal set of generators are incomparable with respect to divisibility. Since we know that any bounded subset of $P_{I}$ has an infimum and supremum in $P_{I}$ it follows that $A$ is a crosscut in $P_{I}$. Moreover, it follows that the crosscut complex $\Gamma\left(P_{I}, A\right)$ is the Buchberger complex $\mathfrak{B} \mathfrak{u}(I)$. Therefore, by Lemma 1.11 and the crosscut theorem [3, Theorem 10.8], it follows that $\mathfrak{B u}(I)$ is contractible.

Set $\Delta=\mathfrak{B u}(I)$, and let $m$ be some monomial such that $\Delta[m]$ is nonempty. Then $\Delta[m]$ consists of all subsets $F \in \mathfrak{B u}(I)$ of $\mathcal{G}(I)$ such that $\mathbf{m}_{F}$ divides $m$. Thus, $F \in \Delta[m]$ if and only if $\mathbf{m}_{F}$ divides $m$, and there is no $u \in \mathcal{G}(I)$ that properly divides $\mathbf{m}_{F}$. Let $J_{m}$ be the monomial ideal generated by all $u \in \mathcal{G}(I)$ such that $u$ divides $m$. Then, $F \in \Delta[m]$ if and only if $F \subseteq \mathcal{G}\left(J_{m}\right)$, and there is no $u \in \mathcal{G}\left(J_{m}\right)$ that properly divides $\mathbf{m}_{F}$. This implies that $F \in \Delta[m]$ if and only if $F \in \mathfrak{B u}\left(J_{m}\right)$, and hence, $\Delta[m]=\mathfrak{B u}\left(J_{m}\right)$. But, then, by the first part of the proof, it follows that $\Delta[m]$ is contractible and hence acyclic.

Thus, we have verified the conditions from Proposition 1.1 for $\Delta=\mathfrak{B} \mathfrak{u}(I)$, and hence, $\mathfrak{B} \mathfrak{u}(I)$ supports a cellular resolution of $I$.

We call the complex $\mathcal{F}_{\mathfrak{B} \mathfrak{u}(I)}$ supported on the Buchberger complex $\mathfrak{B u}(I)$ the Buchberger resolution of the monomial ideal $I$. Next, we characterize when the Buchberger resolution is minimal.

Proposition 1.14. Let I be a monomial ideal and $\mathfrak{B u}(I)$ its Buchberger complex. Then the Buchberger resolution is a minimal free resolution of $I$ if and only if, whenever $F, G \subset \mathcal{G}(I)$ are such that $\mathbf{m}_{F}=\mathbf{m}_{G}$, then there is a monomial $u \in \mathcal{G}(I)$ such that $u$ properly divides $\mathbf{m}_{F}=\mathbf{m}_{G}$. 
Proof. " $\Leftarrow$." Let us assume by contradiction that the Buchberger resolution is not minimal. This means that there are $F, G \in \mathfrak{B} \mathfrak{u}(I)$ and $G \subset F$ such that $\mathbf{m}_{F}=\mathbf{m}_{G}$. By hypothesis, there is a monomial $u \in \mathcal{G}(I)$ such that $u$ properly divides $\mathbf{m}_{F}=\mathbf{m}_{G}$, that is, $G, F \notin \mathfrak{B u}(I)$, a contradiction.

" $\Rightarrow$." Assume that the Buchberger resolution is a minimal free resolution of $I$. Let $F, G \in \mathfrak{B u}(I)$ be such that $\mathbf{m}_{F}=\mathbf{m}_{G}$. If $F \subseteq G$ or $G \subseteq F$, then Proposition 1.2 implies that $F=G$, and we are done. Thus, we can assume that $F$ and $G$ are incomparable with respect to inclusion. Thus, we can choose $m_{\alpha} \in F \backslash G$ and $m_{\beta} \in G \backslash F$. Then, $\left\{m_{\alpha}, m_{\beta}\right\} \in \mathfrak{B u}(I)$ for all $m_{\beta} \in G$, since otherwise there exists $u \in \mathcal{G}(I)$ with

$$
\left.u\right|_{p} \operatorname{lcm}\left(m_{\alpha}, m_{\beta}\right) \mid \mathbf{m}_{F}
$$

and $F \notin \mathfrak{B u}(I)$, a contradiction. Therefore, $\left\{m_{\alpha}\right\} \cup G \in \mathfrak{B} \mathfrak{u}(I)$. Since the resolution is minimal and $\mathbf{m}_{G}=\mathbf{m}_{\left\{m_{\alpha}\right\} \cup G}$, one has $G$ and $\left\{m_{\alpha}\right\} \cup G$ are not faces in $\mathfrak{B} \mathfrak{u}(I)$, a contradiction.

Now we return to the monomial ideal from Example 1.9 and explicitly construct the Buchberger resolution. Moreover, one can easily check or use Proposition 1.14 in order to prove that the resolution is minimal.

Example 1.15. Let

$$
I=\left(x_{1}^{2}, x_{2}^{2}, x_{3}^{2}, x_{1} x_{3}, x_{2} x_{4}\right) \subseteq \mathbb{k}\left[x_{1}, x_{2}, x_{3}, x_{4}\right] .
$$

Then, counting faces in Figure 1 provides

$$
0 \longrightarrow S^{2} \longrightarrow S^{7} \longrightarrow S^{9} \longrightarrow S^{5} \longrightarrow I \longrightarrow 0
$$

as its minimal free resolution over $S$. Moreover, the differentials are easily written out by homogenizing the simplicial differential (see [1], for instance).

For a monomial $m \in L_{I}$, we say that $m$ is a Buchberger degree if there is no $u \in \mathcal{G}(I)$ that properly divides $m$. If $m$ is a Buchberger degree for $I$, then we denote by $B_{m}(I)$ the poset of all subsets $A$ of $[n]:=\{1, \ldots, n\}$ such that, for some $m^{\prime} \in(1, m)$, the set $A$ is the set of indices $i \in[n]$ such that the exponents of $x_{i}$ in $m^{\prime}$ and $m$ coincide. 
Corollary 1.16. Let $I$ be a monomial ideal and $m \in L_{I}$. Then either $\beta_{i, m}=0$ if $m$ is not a Buchberger degree or

$$
\beta_{i, m}=\operatorname{dim}_{\mathbb{k}} \widetilde{H}_{i-1}\left(B_{m}(I) ; \mathbb{k}\right) .
$$

Proof. By Proposition 1.14, we know that $\beta_{i, m}=0$ if $m$ is not a Buchberger degree. Assume $m$ is a Buchberger degree. Consider the map $f:(1, m) \rightarrow B_{m}(I)$ that sends a monomial $m^{\prime}$ to the set of indices $i$ for which the exponents of $x_{i}$ in $m$ and $m^{\prime}$ coincide. For any $A \in B_{m}(I)$, the lower fiber $f^{-1}\left(B_{m}(I)_{\leq A}\right)$ consists of all $m^{\prime} \in(1, m)$ for which the set of indices such that the exponents of $x_{i}$ in $m$ and $m^{\prime}$ coincide is a subset of $A$. Since $A \neq[n]$, it follows that the $1 \mathrm{~cm}$ of all elements of $f^{-1}\left(B_{m}(I)_{\leq A}\right)$ is an element of $(1, m)$. Hence, the fiber $f^{-1}\left(B_{m}(I)_{\leq A}\right)$ has a unique maximal element given by this $\mathrm{lcm}$. Thus, its order complex is a cone, and hence, the fiber is contractible. Then the Quillen fiber lemma says that $(1, m)$ and $B_{m}(I)$ are homotopy equivalent (see [9], [12, Theorem 5.2.1]). In particular, they have the same reduced homology. Since

$$
\beta_{i, m}=\operatorname{dim}_{\mathbb{k}} \widetilde{H}_{i-1}((1, m) ; \mathbb{k}),
$$

the result follows.

Note that, if $m$ is a monomial on $n$ variables $x_{1}, \ldots, x_{n}$, then $B_{m}(I)$ is a subset of the Boolean lattice $2^{[n]}$ on $[n]$. Indeed, $B_{m}(I) \cup\{\emptyset,[n]\}$ is a lattice whose join operation coincides with the union of sets. It is atomic, and its atoms are images of the generators of $I$ dividing $m$. Now Corollary 1.16 shows that, for a monomial $m$ on $n$ variables, the Betti number $\beta_{i, m}$ is bounded from above by the maximal rank of the $(i-1)$ st homology of an atomic join sublattice of the Boolean lattice on $n$ elements. It is also worthwhile to study what can be said about the poset of all $m$ that are Buchberger degrees. Does this poset have an interesting structure? By Corollary 1.16, this question relates to the even more challenging poset of all $m$ for which $\beta_{i, m}$ is nonzero for some $i$. A study of this poset was initiated in [11, Section 8], where first results can be found.

2. Minimality and relation to the Scarf complex. In this section, we are interested in determining the connections between the Buchberger complex and the Scarf complex and the related question 
of when the Buchberger complex defines a minimal free resolution. As before, let $I$ be a monomial ideal with minimal monomial generating set

$$
\mathcal{G}(I)=\left\{m_{1}, \ldots, m_{r}\right\} .
$$

We recall the definition of the Scarf complex.

Definition 2.1. The Scarf complex $\mathfrak{S c}_{I}$ of $I$ is the collection of all subsets of $\mathcal{G}(I)$ whose least common multiple is unique:

$$
\mathfrak{S c}_{I}=\left\{\sigma \subseteq\{1, \ldots, r\}: \mathbf{m}_{\sigma}=\mathbf{m}_{\tau} \Longrightarrow(\sigma=\tau)\right\} .
$$

We call the complex $\mathcal{F}_{\mathfrak{S c}(I)}$ of free $S$-modules, supported on the Scarf complex $\mathfrak{S c}(I)$, the algebraic Scarf complex of the monomial ideal $I$.

Note that, in general, $\mathcal{F}_{\mathfrak{S c}(I)}$ is not a free resolution of $I$ (see details below).

The following remark clarifies the connection between the Scarf complex, the Buchberger graph and the Buchberger complex.

\section{Remark 2.2.}

(i) It is easily seen that $\mathfrak{S c}_{I}^{\langle 1\rangle} \subseteq \mathfrak{B u} \mathfrak{G}(I)$, but the converse does not hold in general.

(ii) One has that $\mathfrak{S c}_{I} \subseteq \mathfrak{B u}(I)$. Indeed, let us assume by contradiction that $F \in \mathfrak{S c}_{I}$ and $F \notin \mathfrak{B u}(I)$. Then, there must be a monomial $u \in \mathcal{G}(I)$ such that $\left.u\right|_{p} \mathbf{m}_{F}$. But, in this case, $\operatorname{lcm}\left(u, \mathbf{m}_{F}\right)=\mathbf{m}_{F}$, that is, $F \notin \mathfrak{S c}_{I}$, a contradiction.

We can characterize the minimality of the Buchberger resolution in terms of the Scarf complex.

Proposition 2.3. Let I be a monomial ideal in $S$. The following are equivalent:

(a) $\mathcal{F}_{\mathfrak{B} \mathfrak{u}(I)}$ is a minimal resolution of $I$.

(b) The Scarf complex $\mathfrak{S c}(I)$ and the Buchberger complex $\mathfrak{B} \mathfrak{u}(I)$ coincide. 
Proof. (a) $\Rightarrow$ (b). Let $\mathcal{F}_{\mathfrak{B} \mathfrak{u}(I)}$ be a minimal free resolution. Assume that there is a face $F \in \mathfrak{B u}(I)$ which is not in the Scarf complex $\mathfrak{S c}(I)$. The latter implies that there is a $G \subseteq \mathcal{G}(I)$ such that $\mathbf{m}_{F}=\mathbf{m}_{G}$. By the minimality of the resolution, Proposition 1.14, we obtain that there is a monomial $u \in \mathcal{G}(I)$ which properly divides $\mathbf{m}_{F}$. But this is a contradiction with $F \in \mathfrak{B u}(I)$.

(b) $\Rightarrow$ (a). If $\mathfrak{S c}_{I}=\mathfrak{B u}(I)$, it is clear that the least common multiple of the monomials from each face of $\mathfrak{B u}(I)$ is unique. The statement follows.

Since it is known [6] that the Scarf complex of a monomial ideal $I$ defines a free resolution of $I$ if and only if it defines a minimal free resolution of $I$, the following corollary is now straightforward.

Corollary 2.4. Let I be a monomial ideal in $S$. If $\mathcal{F}_{\mathfrak{B} \mathfrak{u}(I)}$ is a minimal resolution of $I$, then $\mathcal{F}_{\mathfrak{S c}(I)}$ is a minimal resolution of $I$.

The following example shows that the converse does not hold, in general (see [8, Theorem 5.3] for more details on when the Scarf complex supports a minimal free resolution).

Example 2.5. Let $I=(x a, y b, z c, x y z)$ be a monomial ideal in the polynomial ring $k[x, y, z, a, b, c]$. Since $I$ is a squarefree monomial ideal,

$$
\mathfrak{B u}(I)=\langle\{x a, y b, z c, x y z\}\rangle,
$$

so $\mathcal{F}_{\mathfrak{B} \mathfrak{u}(I)}$ is the Taylor resolution, while the Scarf complex is

$$
\mathfrak{S c}(I)=\langle\{x a, y b, x y z\},\{x a, z c, x y z\},\{y b, z c, x y z\}\rangle,
$$

since $\operatorname{lcm}(x a, y b, z c, x y z)=\operatorname{lcm}(x a, y b, z c)$. One may check that $\mathcal{F}_{\mathfrak{S c}(I)}$ is a resolution and therefore is minimal.

A class of monomial ideals for which the algebraic Scarf complex is a minimal resolution is that of generic monomial ideals (see [1, Theorem 3.2] and [6, Theorem 6.13]).

Definition 2.6. A monomial ideal $I$ is generic if, whenever two distinct minimal generators $m, m^{\prime} \in \mathcal{G}(I)$ have the same positive (nonzero) 
degree in some variable, a third generator $m^{\prime \prime} \in \mathcal{G}(I)$ strictly divides their least common multiple $\operatorname{lcm}\left(m, m^{\prime}\right)$.

One may note that every strongly generic ideal is generic. This implies that the algebraic Scarf complex is a minimal free resolution for strongly generic ideals.

Next we derive a slight generalization of results from [6, Theorem 6.26]. For its formulation, we write $\mathfrak{m}$ for the ideal $\left(x_{1}, \ldots, x_{n}\right)$ in $S$ and $\mathfrak{m}^{\mathbf{u}+1}$ for the ideal

$$
\left(x_{1}^{u_{1}+1}, \ldots, x_{n}^{u_{n}+1}\right),
$$

where $\mathbf{u}=\left(u_{1}, \ldots, u_{n}\right)$ is an $n$-tuple of nonnegative integers.

Proposition 2.7. Fix a vector

$$
\mathbf{u}=\left(u_{1}, \ldots, u_{n}\right)
$$

of nonnegative integers and an ideal

$$
I \subseteq \mathbb{k}\left[x_{1}, \ldots, x_{n}\right]
$$

generated by monomials dividing $\mathbf{x}^{\mathbf{u}}$. Set

$$
\bar{I}=I+\mathfrak{m}^{\mathbf{u}+\mathbf{1}} M,
$$

where $M$ is a monomial with support in

$$
\mathbb{k}\left[x_{n+1}, \ldots, x_{m}\right], \quad m>n .
$$

If I is generic, then $\mathcal{F}_{\mathfrak{B} \mathfrak{u}(\bar{I})}$ is a minimal free resolution of $\bar{I}$. Moreover, $\mathcal{F}_{\mathfrak{S c}(\bar{I})}$ is a minimal free resolution of $\bar{I}$.

In order to prove this result, we need the following lemma.

Lemma 2.8. Let

$$
I \subseteq S=\mathbb{k}\left[x_{1}, \ldots, x_{n}\right]
$$

be a monomial ideal. The following are equivalent:

(a) $\mathfrak{S} \mathfrak{c}(I)=\mathfrak{B} \mathfrak{u}(I)$;

(b) If $F \notin \mathfrak{S c}(I)$ there is a monomial $w \in I$ which properly divides $\mathbf{m}_{F}$. 
Proof. (a) $\Rightarrow$ (b). Let us assume that $\mathfrak{S c}(I)=\mathfrak{B u}(I)$, and let $F \notin \mathfrak{S c}(I)$, that is, $F \notin \mathfrak{B u}(I)$. Therefore, there is a monomial $w \in \mathcal{G}(I)$ which properly divides $\mathbf{m}_{F}$.

(b) $\Rightarrow$ (a). By using Remark 2.2 (ii), we only have to prove that $\mathfrak{B u}(I) \subseteq \mathfrak{S} \mathfrak{c}(I)$. Let us assume by contradiction that there is an $F \in \mathfrak{B} \mathfrak{u}(I)$ such that $F \notin \mathfrak{S c}(I)$. By our assumption, since $F \notin \mathfrak{S c}_{I}$, there is a monomial $w \in I$ which properly divides $\mathbf{m}_{F}$. In particular, there is a monomial $w^{\prime} \in \mathcal{G}(I)$ which properly divides $\mathbf{m}_{F}$, which implies $F \notin \mathfrak{B} \mathfrak{u}(I)$, a contradiction.

Proof of Proposition 2.7. By Proposition 2.3 and Lemma 2.8, we only need to show that, if $F \notin \mathfrak{S c}(\bar{I})$, there is a monomial $w \in \bar{I}$ which properly divides $\mathbf{m}_{F}$. The proof of this statement is identical to the implication [6, Theorem 6.26] "(a) $\Rightarrow(\mathrm{g})$." We recall it here for the sake of completeness.

Let $F \notin \mathfrak{S c}(\bar{I})$, that is, there is a $G \subseteq \mathcal{G}(\bar{I})$ such that $\mathbf{m}_{F}=\mathbf{m}_{G}$. Let us assume that $F$ is maximal with respect to the inclusion among the subsets of $\mathcal{G}(\bar{I})$ with the same label $\mathbf{m}_{F}$. Therefore, there is some $m^{\prime} \in F$ such that $\mathbf{m}_{F}=\mathbf{m}_{F \backslash\left\{m^{\prime}\right\}}$. If $\operatorname{supp}\left(\mathbf{m}_{F}\right)=\operatorname{supp}\left(\mathbf{m}_{F \backslash\left\{m^{\prime}\right\}}\right)$, then $\left.m^{\prime}\right|_{p} \mathbf{m}_{F}$, which ends the proof. Here, for a monomial $m=$ $x_{1}^{a_{1}} \cdots x_{n}^{a_{n}}, \operatorname{supp}(m)=\left\{x_{i}: a_{i} \neq 0\right\}$. Therefore, we assume that $\operatorname{supp}\left(\mathbf{m}_{F}\right) \neq \operatorname{supp}\left(\mathbf{m}_{F \backslash\left\{m^{\prime}\right\}}\right)$. Since $\mathbf{m}_{F}=\mathbf{m}_{F \backslash\left\{m^{\prime}\right\}}$, there is a monomial $m^{\prime \prime} \in F, m^{\prime \prime} \neq m^{\prime}$, and a variable $x_{k} \in \operatorname{supp}\left(m^{\prime \prime}\right)$ such that $\operatorname{deg}_{k}\left(m^{\prime}\right)=\operatorname{deg}_{k}\left(m^{\prime \prime}\right)>0$. Here we denote the exponent of the variable $x_{k}$ in the monomial $m$ by $\operatorname{deg}_{k}(m)$. Since $m^{\prime \prime} \neq m^{\prime}$, we must have that neither $m^{\prime}$ nor $m^{\prime \prime}$ are in $\mathcal{G}\left(\mathfrak{m}^{\mathbf{u}+\mathbf{1}} M\right)$. Therefore, $m^{\prime}, m^{\prime \prime} \in \mathcal{G}(I)$ and, since $I$ is generic, there is a monomial $w \in \mathcal{G}(I)$ such that $\left.w\right|_{p} \operatorname{lcm}\left(m^{\prime}, m^{\prime \prime}\right)$. Since $\operatorname{lcm}\left(m^{\prime}, m^{\prime \prime}\right) \mid \mathbf{m}_{F}$, the statement follows.

The preceding results show that the Buchberger complex is an interesting object and encodes very well the combinatorics and algebra of monomial ideals. Nevertheless, there is an alternative object which equally generalizes well the Buchberger graph from [5] in the situation where it can be embedded with triangular cells. For a monomial ideal $I$ and its Buchberger graph $\mathfrak{B u} \mathfrak{G}(I)$, let $\mathrm{Cl}(\mathfrak{B} \mathfrak{u} \mathfrak{G}(I))$ be the simplicial complex whose simplices are the subsets of $\mathcal{G}(I)$ that induce a clique in 
$\mathfrak{B} \mathfrak{u} \mathfrak{G}(I)$; this construction is also known as the clique complex or Rips complex of $\mathfrak{B} \mathfrak{u} \mathfrak{G}(I)$.

We conjecture the following.

Conjecture 2.9. For a monomial ideal I the clique complex $\mathrm{Cl}(\mathfrak{B u G}(I))$ of the Buchberger graph $\mathfrak{B} \mathfrak{u} \mathfrak{G}(I)$ is contractible.

In particular, if the conjecture holds true, then simple reasoning shows that $\mathrm{Cl}(\mathfrak{B u G}(I))$ also supports a minimal free resolution of $I$. Clearly, $\mathfrak{B} \mathfrak{u}(I) \subseteq \mathrm{Cl}(\mathfrak{B u} \mathfrak{G}(I))$, and therefore the construction, will not yield more cases where we can construct a cellular minimal free resolution. Nevertheless, we believe the conjecture seems appealing from a combinatorial point of view. The conjecture is supported by Theorem 1.6 in the three variable cases and many experiments.

Acknowledgments. The authors thank Lukas Katthän for pointing out an error in the formulation of Corollary 2.4 in a previous version of this paper.

\section{REFERENCES}

1. D. Bayer, I. Peeva and B. Sturmfels, Monomial resolutions, Math. Res. Lett. 5 (1998), 31-46.

2. D. Bayer and B. Sturmfels, Cellular resolution of monomial modules, J. reine angew. Math. 502 (1998), 123-140.

3. A. Björner, Topological methods, in Handbook of combinatorics, II, R.L. Graham, M. Grötschel and L. Lovász, eds., Elsevier, Amsterdam, 1995.

4. V. Gasharov, I. Peeva and V. Welker, The lcm-lattice in monomial resolutions, Math. Res. Lett. 6 (1999), 521-532.

5. E. Miller and B. Sturmfels, Monomial ideals and planar graphs, in Applied algebra, algebraic algorithms and error-correcting codes, Lect. Notes Comp. Sci. 1719 (1999), 19-28.

6. _ Combinatorial commutative algebra, Grad. Texts Math., Springer, 2005.

7. I. Peeva, Graded syzygies, Alg. Appl. 14, Springer, London, 2011.

8. I. Peeva and M. Velasco, Frames and degenerations of monomial resolutions, Trans. Amer. Math. Soc. 363 (2011), 2029-2046.

9. D. Quillen, Homotopy properties of the poset of non-trivial p-subgroups of a group, Adv. Math. 28 (1978), 74-95.

10. D. Taylor, Ideals generated by an R-sequence, Ph.D. dissertation, University of Chicago, Chicago, 1966. 
11. A. Tchernev and M. Varisco, Modules over categories and Betti posets of monomial ideals, Proc. Amer. Math. Soc. 143 (2015), 5113-5128.

12. M.L. Wachs, Poset topology: Tools and applications, Lect. Notes IAS/Park City Graduate Summer School Geom. Combin., 2004.

University Politehnica of Bucharest, Faculty of Applied Sciences, Splaiul Independenţei, No. 313, 060042, Bucharest, Romania and Simion Stoilow Institute of Mathematics of the Romanian ACAdemy, Research group of the Project PD-3-0235, P.O. Box 1-764, Bucharest 014700, Romania

Email address: olteanuandageorgiana@gmail.com

Fachbereich Mathematik und Informatik, Philipps-Universität Marburg, 35032 Marburg, Germany

Email address: welker@mathematik.uni-marburg.de 